\title{
Differences between serious and nonserious patient safety incidents in the largest hospital district in Finland
}

\author{
Jämsä, J.O.
}

2018

Jämsä , J O , Palojoki , S H , Lehtonen , L \& Tapper , A-M 2018 , ' Differences between serious and nonserious patient safety incidents in the largest hospital district in Finland ', Journal of healthcare risk management, vol. 38 , no. 2 , pp. $27-35$. https://doi.org/10.1002/jhrm.21310

http://hdl.handle.net/10138/325863

https://doi.org/10.1002/jhrm.21310

unspecified

acceptedVersion

Downloaded from Helda, University of Helsinki institutional repository.

This is an electronic reprint of the original article.

This reprint may differ from the original in pagination and typographic detail.

Please cite the original version. 


\section{Differences between Serious and Non-Serious Patient Safety Incidents in the Largest Hospital District in Finland}

Authors:

Jämsä Juho Olavi, MB, BA (Cognitive Science) ${ }^{1}$

Palojoki Sari Hannele, MSc (health care), $\mathrm{PhD}$ (health informatics) ${ }^{2}$

Lehtonen Lasse $\mathrm{MD}, \mathrm{PhD}^{3}$

Tapper Anna-Maija MD, $\mathrm{PhD}^{4}$

Affiliations:

1. University of Helsinki, P.O. Box 63 FIN-00014, University of Helsinki, Finland

2. Group Administration, Hospital District of Helsinki and Uusimaa, P.O. Box 100, FIN00029 HUS, Finland

3. Group Administration, Hospital District of Helsinki and Uusimaa, P.O. Box 100, FIN00029 HUS, Finland

4. Hyvinkää Hospital, University of Helsinki and Helsinki University Hospital, P.O. Box 100, FIN-00029, HUS, Finland

Conflicts of Interest and Source of Funding: None declared 
Corresponding author:

Jämsä Juho

tel: +358405485166

e-mail: juho.jamsa@helsinki.fi

\section{About the Authors}

Jämsä J, MB, BA, works as an airline pilot for Finnair as first officer in A350 and is also studying for an MD at the University of Helsinki. Palojoki S, MSc, PhD is Chief of Patient Safety at Helsinki and Uusimaa University Hospital District hospitals in Finland. Lehtonen L, MD, PhD is the Chief Administrative Physician at Helsinki and Uusimaa University Hospital District and Professor of Health Law at University of Helsinki.

Tapper A-M, MD, PhD, MBA is Medical Director at Hyvinkää Hospital at Helsinki and Uusimaa University Hospital District and works for the University of Helsinki as a researcher in patient safety. 


\section{Abstract}

Objectives: To determine if and in what ways serious patient safety incidents differ from non-serious patient safety incidents.

Methods: Statistical analysis was performed on patient safety incident reports that were reported in 2015 in Finland's largest hospital district (Helsinki and Uusimaa, HUS). Reports were divided into two groups: non-serious incidents and serious incidents. Differences between groups were studied from several types of categorically divided information.

Results: Of the total amount of reports (15 863) $1 \%$ were serious incidents (175). Serious and non-serious incidents differed significantly from each other. Serious incidents concerning laboratory, imaging or medical equipment were more common. On the other hand incidents concerning medication, infusion, blood transfusion, were less frequent. In serious incidents the proportion of doctors reporting was greater and contributing factors were better recognized, the most common of them being working of procedures.

Conclusions: In the future, special attention should be given to the particular aspects of serious patient safety incidents, such as safe use of medical equipment, training and handling of procedures. Root cause analysis is an effective way to handle serious incidents and enables the prevention of their reoccurrence. However, a systematic follow of the root cause analysis should be developed. 
MeSH keywords: patient safety, patient harm, medical errors, risk management 


\section{Introduction}

Scientific research on patient safety began in the 1980s, especially in the United States (1). In Finland, research on this subject began vigorously only in the early 2000s. In 2009, the Ministry of Social Affairs and Health in Finland announced for the first time a national patient safety strategy and in 2011 a plan for quality management and for ensuring patient safety was published and implemented in Finnish legislation (2, 3). Also currently training programs for health care professionals on patient safety (4) and a nationwide reporting system for incidents and near miss events have been implemented in the health care system.

A patient safety incident (safety incident) is an adverse event compromising patient safety that could have or did lead to patient harm. Most of these are non-serious patient safety incidents (non-serious incident). However, serious patient safety incidents (serious incident) are also reported. A serious incident is an event that leads to substantial, serious or permanent harm to patient, causes serious danger to the life or safety of the patient, or is a patient safety incident concerning a large group of patients. Serious harm is a consequence from an event that leads to a patient's death, commencement or continuation of hospitalization, serious impairment, disability or an event that is life-threatening $(5,6)$. Serious incidents form a small but significant fraction of safety incidents and it is important to study their nature and prevalence.

The aspects of human error, especially from the view of human factors, are widely covered by Reason (7). He divides human action into routine processes, more unfamiliar situations and novel situations. In each situation certain type of errors are expected: slips and lapses; rule-based mistakes; and knowledge based mistakes respectively. 
The current scientific view on human error emphasizes the system approach, according to which working environments and organizational processes are such that errors are expected $(8,9,10)$. In addition, working procedures at all levels of the organization drift towards procedures that are more likely to cause errors $(9,10)$. Therefore, organizations must have processes through which procedures are monitored, incidents are reacted to and lessons are learned from them and finally, future events are anticipated (9).

Improving patient safety is essential from both humane and economical perspectives. In Finland, the Finnish Patient Insurance Centre handles all personal injuries that occur in connection with healthcare activities. It compensates annually about 2200 patient injuries and their costs are about 41 million euros (11). Much of the costs of medical errors are, however, covered either by health care units in their extra costs or by patients themselves due to prolonged disabilities and suffering.

\section{Methods}

The purpose of this study was to determine if and in what ways serious incidents differ from non-serious incidents. An additional objective was to identify the nature and number of both types of incidents.

All Finnish hospital districts, together more than 144000 health care professionals, use a common system for reporting patient safety incidents (HaiPro). HaiPro reporting program has been developed by Awanic $\AA$ and has been used since 2009. Finland is divided into 19 hospital districts. Helsinki and Uusimaa District (HUS) is the largest hospital district in Finland and is further divided into five regionally determined hospital areas. HUS also runs the largest academic teaching hospital in Finland (Helsinki University Hospital), which covers all medical specialties in tertiary care and emergency services within the district. The 
number of patient safety reports continues to rise annually. Data consisted of HaiPro reports generated in the HUS district in 2015. (4)

Patient safety at HUS is promoted through the principles of risk assessment. Risk assessment is the process through which organizations develop an understanding of the risks they face (12). The Patient Safety Development Program at HUS is coordinated by the Chief of Patient Safety and e.g., the patient safety surveillance is guided by the steering committee which monitors patient safety data on a regular basis.

\subsection{HaiPro reporting system}

The HaiPro reporting form is an easy-to-fill, e-form and can be accessed from the HUS intranet homepage by anyone who works for HUS. It can be done anonymously and employees are strongly encouraged to report incidents. The HaiPro system is not yet available for patients (4). A separate procedure for reporting and analyzing severe patient safety incidents has been in use since 2013. In the electronic HaiPro form, several types of categorically divided information are selectable from dropdown menus. In this study, the HaiPro categories are italicized. The administrative unit of the incident and of the employee, occupation, time of incident, place of incident, nature of incident (actual or near miss) and finally type of incident are selected. In addition, what happened, the consequences of the incident, contributing factors and what could be done to avoid similar incidents in the future are recorded. After submission, the report is automatically forwarded for analysis by a doctor-nurse pair that is responsible for patient safety in the given unit.

Analysis begins by specifying and by changing the type of incident, which can be specified from fifteen categories and several subcategories. Additional information can be requested from the person who filed the report. The degree of harm is specified and the consequences 
for the unit are selected. Next, the level of risk is determined by assessing the likelihood of reoccurrence and the severity of the consequences using the HaiPro risk matrix (Figure 1). Control of the situation and immediate actions are also specified. The possible contributing factors are specified (ten categories, several subcategories). Finally, the preventive action is specified (four categories, several subcategories). The handling of a safety incident report is illustrated in Figure 2.

The analysis of serious incidents differs from the procedure depicted earlier. A separate root cause analysis (RCA) is coordinated by a serious incident analyst. If the degree of harm is specified as serious or the incident has a risk level of IV or V, the report is automatically directed to the serious incident analyst who then confirms the serious incident procedure or returns the report to the regular safety incident analysis with explanation for the return. If needed and if contact information is available, the serious incident analyst can request for additional information from the person who filed the report. All serious incidents are transferred to a higher level of organization and discussed in a multi-professional group led by the serious incident coordinator. The chain of events is analyzed using the Ishikawa procedure (13). With this method, particular causes that lead to the effect are identified. For each effect, the actions promoting patient safety and the persons responsible for the actions are defined and a deadline is given. After the RCA, the person marked as responsible for the actions required receives an email about the task. The serious incident report is marked as completed after all the necessary actions are performed.

\subsection{Data and Statistical Methods}

HaiPro reports were divided into two groups: non-serious incidents and serious incidents. Groups were created 2016/3/3. To determine how non-serious incidents and serious incidents differed in their nature, the number of reports in each category (e.g. type of 
incident: medical equipment or its operation) was compared between the groups using a Chisquared test. The results from the main categories are the focus of this study. A cut-off $p$ value of 0.05 was used to evaluate statistical significance. If the number of reports was too small to perform the test reliably, they were excluded (N/A, not available)

\section{Results}

\subsection{HaiPro reports in HUS district}

In the year 2015 there were in total 15863 HaiPro reports made in the HUS district. Of these, 15688 were non-serious incidents and $175(1 \%)$ were serious incidents.

In Hospital area 1 (including university hospital), the number of non-serious incidents was 10406 and serious incidents 100. The range of serious incident percentages between Hospital area 1 units was $0.2 \%$ to $3.8 \%$. The range of percentages between Hospital areas was $0.7 \%$ to $2.4 \%$. The greatest percentage of serious incidents in Hospital area $1(\mathrm{n}=38$, $3.8 \%$ ) was reported in the unit including operating rooms, intensive care and pain management. (Table 1.)

\subsection{Non-serious incidents, serious incidents and their differences}

According to this study, there are statistically significant differences in the nature between serious and non-serious incidents. It is noteworthy, that serious incidents were more commonly concerned with laboratory tests and medical equipment and that the most common contributing factor was handling of procedures.

The greatest proportion of the serious incidents happened in patient rooms (non-serious 4284 [27\%], serious 60 [34\%], $\mathrm{p}<0.05$ ) and operating rooms (non-serious 801 [5\%], serious 
$26[15 \%], \mathrm{p}<0.05)$. In laboratory the proportion of incidents between groups was equal (non-serious 1638 [10\%], serious 16 [9\%], p > 0.05). Regarding weekdays, there were no differences between groups. In both groups, errors in working days were more common than in Saturdays and Sundays. (data not shown)

Doctors filed a greater proportion of serious incidents (non-serious 763 [5\%], serious 28 [16 $\%], \mathrm{p}<0.05)$. However, in both groups nurses filed the reports most often (non-serious 11388 [73\%], serious 107 [61\%], p < 0.05). Incidents that actually happened were reported more often in serious incidents (non-serious 9037 [58 \%], serious 139 [79\%], p < 0.05). Near miss reporting was more common among nurses and other personnel. In serious incidents the degree of harm was most commonly serious (33\%) (data not shown).

The summary of incident types is shown in Table 2 . The most common serious incidents were Laboratory, imaging or other tests (non-serious 2754 [18\%], serious 41 [23\%], p < 0.05 ) followed by other treatment or monitoring (non-serious 1282 [8\%], serious 26 [15 $\%$ ], $\mathrm{p}<0.05$ ) and medical equipment or its operation (non-serious 880 [6\%], serious 23 [13 $\%], \mathrm{p}<0.05)$. The most common non-serious incident was Medication, infusion, blood transfusion, contrast medium (non-serious 5900 [38\%], serious 25 [14\%], p < 0.05). It was the fourth most common among serious incidents.

As for the specific type of incident within serious incidents the most important were: sample taken from the wrong patient (Laboratory, imaging or other tests), monitoring neglected or the need for it not recognized (other treatment or monitoring) and equipment malfunction (medical equipment or its operation) (data not shown).

A greater proportion of the consequences for the unit in serious incidents were extra work, minor extra treatment (non-serious 8084 [52\%], serious 118 [67\%], $<$ < 0.05) and harm to 
unit image (non-serious 4339 [28\%], serious 73 [42\%], p < 0.05). In addition, in serious incidents, a clear difference was seen in longer stay of care (non-serious 609 [4 \%], serious $49[28 \%], \mathrm{p}<0.05)$ and extra costs (non-serious 684 [4\%], serious 32 [18\%], p < 0.05).

A summary for contributing factors is shown in Table 3. In serious incidents, not known presented a smaller proportion (non-serious 4001 [26\%], serious 15 [9\%], p < 0.05). On the other hand, the most common contributing factor in serious incidents was handling of procedures (non-serious 2985 [19\%], serious 62 [35\%], p < 0.05). Also more common in serious incidents were training, orientation and skills (non-serious 1212 [8\%], serious 27 [15\%], $\mathrm{p}<0.05$ ) followed by medical device and equipment (non-serious 601 [4\%], serious $23[13 \%], \mathrm{p}<0.05)$.

Proposals for preventive actions differed in each category between groups. Common serious incidents included decision to a higher organizational level (non-serious 380 [2 \%], serious 42 [24\%], p < 0.05), no actions needed (non-serious 1938 [12\%], serious 38 [22\%], p < 0.05 ) and plan a procedure to prevent future incidents (non-serious 640 [4\%], serious 30 [17\%], $\mathrm{p}<0.05$ ). The proportion of inform/discuss about the incident (non-serious 12298 [78\%], serious 72 [41\%], $\mathrm{p}<0.05)$ was significantly smaller in serious incidents but was the greatest category in both groups.

\section{Discussion}

To our knowledge, there are no previous studies in the English literature that focus on the differences between non-serious and serious incidents. Thus, this study brings new and more specific scientific evidence on the nature of patient safety incidents. 
Ruuhilehto et.al. analyzed all safety incidents made with the HaiPro system in Finland from 2007-2009 (14). Regarding non-serious incidents, findings are consistent with our study. However at that time separate RCA for serious incidents was not in use.

The National Health Service England (NHS) publishes quarterly reports from the National Reporting and Learning System (NRLS). Data is available from all safety incidents. Serious incidents are directed to a separate serious incident management process and public data containing only serious incidents is not available. In 2014, the most common type of incident in the NRLS data was patient accident $(19 \%)$. When analyzing the combined number of incidents with degree of harm serious harm and death, the most common type of incident was implementation of care and ongoing monitoring / review (19\%) (15). This is in accordance with our study where other treatment or monitoring was more common in serious incidents.

In Finland, health care professionals receive training for the use of HaiPro. Use of incident reporting systems as such improves patient safety. Furthermore, the training received by health care professionals increases their knowledge about safety culture, which in turn also improves patient safety $(16,17,18)$. Especially with regards to this study, the level of organizational safety culture may also impact the degree of safety incident seriousness (19). In general, health care organizations should specifically work to create a nonpunitive safety culture in order to improve patient safety (20). The number of incident reports in Finland has risen annually, which is indicative of improving patient safety culture and increasing patient safety in general.

According to our study, about $1 \%$ of all safety incidents in HUS are serious, which is in accordance with other studies $(14,15,16)$. However, only about $2 \%$ of patients are involved in a safety incident, when compared to the total number of treated different patients. This 
finding does not correspond to other studies, according to which approximately $10 \%$ of patients are involved in a safety incident (21). This discrepancy may be a sign of underreporting. There are no previous studies in Finland that consider how many of the incidents actually get reported. According to some studies, approximately $20 \%$ of the employees admit omitting a non-serious incident report and about $4 \%$ admit that they have not filed a report after a serious incident $(22,23)$. Incident reporting willingness in Finland should be further studied.

In both groups, nurses had submitted the majority of reports, which is consistent with previous studies $(22,24,25)$. However, the portion of reports submitted by doctors was three times higher in serious incidents. It seems that the employees' willingness to report incidents increases with severity of incidents (22), but to our knowledge there are no studies that try to resolve the differences regarding doctors' willingness to report serious versus non-serious incidents. According to other studies, doctors' willingness to report safety incidents in general is reduced, for example, by insufficient training on patient safety, fear of blame, embarrassment, fear of consequences for their future career or the belief that it is not their responsibility to report others' mistakes $(22,24,25)$. On the contrary, willingness to report increases if the reporting provides a possibility to learn from mistakes (26), feedback is given and reports are kept confidential (27). The increased amount of serious incident reports by doctors may in part arise from the fact that doctors perform more risky procedures and that doctors are legally responsible for patient care (3). In conclusion, it seems that doctors' activity increases when incidents are serious. The RCA Tool developed by the hospital district facilitates a structured process for an analysis of serious cases. This further promotes willingness to report serious incidents, especially when they are handled nonpunitively. This phenomenon is suggested for future research. 
The number of reports in both groups was twice as large on working days compared to reporting on weekends. This is explained at least in part with the corresponding allocation of elective operations on weekdays. In addition, weekday was the only type of data where there were no statistically significant differences between groups.

Harm to unit image was almost twice as common of a consequence for the unit in serious incidents compared to non-serious incidents. This finding may in part reflect undeveloped patient safety culture and employees' beliefs that a serious incident is shameful. If this is the case, employees in a way have not accepted the fact that mistakes can happen anytime and to anyone (7, 8, 9 and 10). Additionally, longer stay of care and extra costs were more common consequences for the unit in serious incidents. According to this study, serious incidents are critical for the institution from the economical perspective. Further, in serious incidents the degree of harm was usually serious. Therefore serious incidents are critical also for the patient from humane perspective. Targeted patient safety training directed decreasing the serious incidents can not only reduce human suffering but also reduce total costs $(16,17$, 18).

Other studies, as well as ours, have found medication-related incidents to be most common $(15,28)$. However, in our study among serious incidents medication-related incidents were only the fourth most common type, while laboratory, imaging or other tests was the most common type of incident. In the NRLS data, "degree of harm by type" categories of severe and death can be combined as one category and then be compared with serious incidents in our study (15). In that case the proportion of medication in the NRLS data also decreased, which is in accordance with our study. In the NRLS data, medical device / equipment is a fairly rare category in general. This is inconsistent with our study, where medical equipment or its operation-related incidents were common (5,6\% of non-serious), especially in the serious incidents $(13,1 \%)$. Further studies are necessary to determine the effectiveness of 
training, safe use of medical equipment and their maintenance since in this study contributing factors such as handling of procedures and training, orientation and skills and medical device and equipment were also more common in the serious incidents.

In the non-serious incidents, contributing factors were not known in one-fourth of the incidents, three times more than in serious incidents. It seems that in serious incidents contributing factors are recognized more effectively. Preventive actions were also more commonly reported. Plan a procedure to prevent future incidents or decision to a higher organizational level were more common in serious incidents. Therefore, the RCA where effects are identified and the actions for promoting patient safety are specified seems to be an appropriate way to handle serious patient safety incidents.

In order to achieve effective and sustainable solutions following a RCA health care organizations should consider for example institutional changes and updates in IT structures. However many smaller solutions together, such as training and counselling can also have an effective and sustainable impact on patient safety. (29)

In our study actions to be done usually included discussions with the persons involved, reminders to the personnel about applicable guidelines or processing the cases in unit meetings. Procedures were also updated and new guidelines created. Additionally, extra training was provided for the personnel or new equipment were purchased. Further studies are necessary to determine if the performed actions have an impact on patient safety. It is noteworthy, that in the HaiPro system the completion of the actions promoting patient safety are not automatically monitored. A system that follows the progression of actions promoting patient safety is required. 
To summarize the findings regarding RCA, our study seems to be in accordance with the current scientific view on the matter; the method is promising but is rarely used to its full potential. In our study the most notable weakness of RCA was the lack of measuring of outcomes. There are also other widely recognized problems with RCA. For example analysis often results in a linear chain of events (who, what) rather than a more realistic systemic view (how) and the causes are expressed too vaguely. Secondly, the group performing RCA is usually a local team rather than a diverse group of expert accident investigators with a range of knowledge from subject matter to human factors and interview techniques. The results of the analysis are also rarely shared with the persons involved, persons that might come across with a similar situation, or with other units and organizations. The purpose of sharing the results, in fact the purpose of the whole RCA method, is to learn from the events and to prevent them from happening again. $(30,31,32)$

One possible solution for the shortcomings of RCA could be the use of the Human Factors Analysis Classification System specifically tailored to the health care industry (32). This method would allow for a standardized approach to identify "why" instead of "who and what". Human and environmental factors would be more thoroughly investigated and a standardized nomenclature would provide the basis for sharing information across organizations.

There are some limitations in this study which are suggested to be taken into consideration. The main limitation is the type of HaiPro data which doesn't represent the actual rate of incidents. However, this study does not seek to describe an incident rate but rather a descriptive analysis of the phenomenon which has seldomly been studied.

\section{Conclusion}


In conclusion, the HaiPro reporting system is increasingly adopted in the Finnish health care system. The systematic incident reporting procedure has been in use for a fairly short time in Finland and the number of reports continues to rise annually. The increasing number of patient safety reports is a sign of improved patient safety culture, which in turn is critical for further improving patient safety. It is likely that not all incidents are reported, so additional effort is necessary to further raise reporting willingness. Doctors' increased activity to report serious incidents should be further studied. In the future, special attention should be given to the particular aspects of serious patient safety incidents, such as safe use of equipment, training and procedures. A system that follows the progression of the actions for promoting patient safety should be developed. 


\section{References}

1. Leape LL. Scope of Problem and History of Patient Safety. Obstet Gyn Clin N Am 2008;35:1-10.

2. Ministry of Social Affairs and Health (Finland). Promoting Patient Safety Together. $\begin{array}{lllll}\text { Finnish } & \text { Patient } & \text { Safety } & \text { Strategy }\end{array}$ https://julkaisut.valtioneuvosto.fi/bitstream/handle/10024/72272/potilasturvallisuus_jul kaisu_2009_3_verkko_UP.pdf?sequence=1. [in Finnish] Accessed April 24, 2017.

3. Ministry of Social Affairs and Health (Finland). Health Care Act (1326/2010). 2010. http://www.finlex.fi/en/laki/kaannokset/2010/en20101326.pdf. Accessed April 24, 2017.

4. Helsinki and Uusimaa Hospital District. Helsinki and Uusimaa Hospital District Patient Safety Plan 2016-2017. http://www.hus.fi/potilaalle/laatu-japotilasturvallisuus/potilasturvallisuushussa/Documents/HUS\%20Potilasturvallisuussuu nnitelma\%202016-17. pdf Accessed April 24, 2017.

5. The Finnish Society for Patient Safety. Investigating Serious Patient Safety Incidents. A Guide for Social Affairs- and Health Care Organisations. 2012. 
http://www.potilasturvallisuusyhdistys.fi/documents/vakavien_opas.pdf. [in Finnish] Accessed April 24, 2017.

6. National Institute for Health and Welfare (Finland). Patient Safety Vocabulary 2007. https://www.thl.fi/documents/10531/102913/potilasturvallisuuden_sanasto_071209.pdf [in Finnish] Accessed April 24, 2017.

7. Reason J. Human Error. New York, NY: Cambridge university press 1990.

8. Reason J. Managing the Risks of Organizational Accidents. Aldershot: Ashgate Publishing Limited. 1997.

9. Hollnagel E. Resilience Engineering in Practice: A Guidebook. Ashgate Publishing Limited. 2010.

10. Dekker S, Pruchnick S. Drifting into failure: theorising the dynamics of disaster incubation. Theor Issues Ergonomics Sci. 2014;15:534-44.

11. Patient Insurance Centre (Finland). Statistics of patient injuries. 2016. http://www.pvk.fi/en/statistics/. Accessed April 24, 2017. 
12. ISO 31000(en): Risk management - principles and guidelines on implementation ISO/TC 262. Geneva, Switzerland: ISO Copyright Office; Nov 15, 2009.

13. Ishikawa, K. What is Total Quality Control? The Japanese Way. New Jersey: Prentice Hall 1985.

14. Ruuhilehto K, Kaila M, Keistinen T, Kinnunen M, Vuorenkoski L, Wallenius J. HaiPro - what was learned from patient safety incidents in Finnish health care units in 2007 to 2009? Duodecim; laaketieteellinen aikakauskirja 2011;127:1033-40. [in Finnish]

15. National patient safety agency. Patient safety incident reports in the NHS: reporting and learning system quarterly data summary. 2015.

http://www.nrls.npsa.nhs.uk/patient-safety-data/. Accessed April 24, 2017.

16. Sax Institute for the Australian Commission on Safety and Quality in Health Care. Evidence check. Patient safety in primary healthcare. 2015. http://www.saxinstitute.org.au/wp-content/uploads/Patient-safety-in-primaryhealthcare.pdf. Accessed April 24, 2017. 
17. Brilli RJ, McClead RE, Crandall WV, Stoverock L, Berry JC, Wheeler TA, Davis JT. A Comprehensive Patient Safety Program Can Significantly Reduce Preventable Harm, Associated Costs, and Hospital Mortality. J Pediatr 2013;163:1638-45.

18. Donnelly LF, Dickerson JM, Goodfriend MA, Muething SE. Improving Patient Safety: Effects of a Safety Program on Performance and Culture in a Department of Radiology. Am J Roentgenol. 2009; 193:165-71.

19. Kline T, Willness C, Ghali W. Determinants of adverse events in hospitals -the potential role of patient safety culture. J Healthc Qual. 2008;30:11-7.

20. Occelli P, Quenon JL, Hubert B, Kosciolek T, Hoarau H, Pouchadon ML, Amalberti Y, Auroy Y, Salmi LR, Sibé M, Parneix P, Michel P. Development of a safety culture: initial measurements at six hospitals in France. J Healthc Risk Manag, 2011;30(4):42-47.

21. de Vries EN, Ramrattan MA, Smorenburg SM, Gouma DJ, Boermeester MA. The incidence and nature of in-hospital adverse events: a systematic review. Qual Saf Health Care. 2008;17:216-23.

22. Smith KS, Harris KM, Potters L, Sharma R, Mutic S, Gay HA, Terezakis S. Physician Attitudes and Practices Related to Voluntary Error and Near-Miss Reporting. J Oncol Pract. 2014;10: 350-57. 
23. Kaldjian LC, Jones EW, Wu BJ, Forman-Hoffman VL, Levi BH, Rosenthal GE. Reporting Medical Errors to Improve Patient Safety: A Survey of Physicians in Teaching Hospitals. Arch Intern Med. 2008;168:40-46.

24. Bagenal J, Sahnan K, Shantikumar S. Comparing the Attitudes and Knowledge Toward Incident Reporting in Junior Physicians and Nurses in a District General Hospital. $J$ Patient Saf. 2016;12: 51-53.

25. Shu Q, Tao HB, Fu J, Zhang RN, Zhou J, Cheng ZH. The Differences Between Doctors' and Nurses' Attitudes Toward Adverse Event Reporting and Assessments of Factors That Inhibit Reporting. American Journal of Medical Quality May-June 2014;29:26263 Available from: American College of Medical Quality, Bethesda, MD, Accessed April 24, 2017.

26. Hewitt T, Chreim S, Forster A. Sociocultural Factors Influencing Incident Reporting Among Physicians and Nurses: Understanding Frames Underlying Self- and PeerReporting Practices. J Patient Saf, 2014;00:1-9.

27. Howell AM, Burns EM, Bouras G, Donaldson LJ, Athanasiou T, Darzi A. Can Patient Safety Incident Reports Be Used to Compare Hospital Safety? Results from a Quantitative Analysis of the English National Reporting and Learning System Data. 
PLoS ONE 2015;10:e0144107 Available from. PLOS, San Francisco, CA, Accessed April 24, 2017.

28. World Health Organization. Summary of the evidence on patient safety: implications for research. 2008. http://www.who.int/patientsafety/information_centre/20080523_Summary_of_the_evid ence_on_patient_safety.pdf. Accessed April 24, 2017.

29. Hettinger AZ, Fairbanks RJ, Hegde S, Rackoff AS, Wreathall J, Lewis VL, Bisantz AM, Wears RL. An evidence-based toolkit for the development of effective and sustainable root cause analysis system safety solutions. J Healthc Risk Manag, 2013;33(2):11-20.

30. Peerally MF, Carr S, Waring J, Dixon-Woods, M. The problem with root cause analysis. BMJ Qual Saf, 2017;26(5):417-422.

31. National Patient Safety Foundation. RCA ${ }^{2}$ : Improving Root Cause Analyses and Actions to Prevent Harm http://c.ymcdn.com/sites/www.npsf.org/resource/resmgr/PDF/RCA2_v2-onlinepub_010816.pdf . Accessed August 20, 2017.

32. Diller T, Helmrich G, Dunning S, Cox S, Buchanan A, Shappell S. The human factors analysis classification system (HFACS) applied to health care. Am J Med Qual, 2014;29(3):181-190. 
Table 1. HaiPro reports in HUS Hospital areas (including Hospital area 1 units separately). Table presents the numbers of safety incidents, non-serious incidents, serious incidents and the percentage of serious incidents in relation to all safety incidents (\%). Information is given from each Hospital area.

\begin{tabular}{|c|c|c|c|c|}
\hline Hospital area 1 & safety incident & non-serious & serious & $\%$ \\
\hline children's and juvenile diseases & 1575 & 1570 & 5 & $0.3 \%$ \\
\hline psychiatry & 1147 & 1132 & 15 & $1.3 \%$ \\
\hline acute & 1027 & 1022 & 5 & $0.5 \%$ \\
\hline head and neck & 1008 & 1004 & 4 & $0.4 \%$ \\
\hline operating rooms, intensive care, pain management & 990 & 952 & 38 & $3.8 \%$ \\
\hline oncology & 878 & 876 & 2 & $0.2 \%$ \\
\hline abdominal & 826 & 822 & 4 & $0.5 \%$ \\
\hline heart and lung & 791 & 782 & 9 & $1.1 \%$ \\
\hline gynecology and labor & 643 & 631 & 12 & $1.9 \%$ \\
\hline rheumatic diseases and plastic surgery & 619 & 617 & 2 & $0.3 \%$ \\
\hline internal medicine and rehabilitation & 561 & 558 & 3 & $0.5 \%$ \\
\hline inflammation & 441 & 440 & 1 & $0.2 \%$ \\
\hline Hospital area 1 , total & 10506 & 10406 & 100 & $1.0 \%$ \\
\hline Hospital area 2 & 1158 & 1143 & 15 & $1.3 \%$ \\
\hline Hospital area 3 & 926 & 919 & 7 & $0.8 \%$ \\
\hline Hospital area 4 & 536 & 532 & 4 & $0.7 \%$ \\
\hline Hospital area 5 & 248 & 242 & 6 & $2.4 \%$ \\
\hline$\underline{\text { HUSLAB }}$ & 2043 & 2000 & 43 & $2.1 \%$ \\
\hline HUS imagining & 277 & 277 & 0 & $0.0 \%$ \\
\hline Other (e.g. administrative, catering, maintenance) & 169 & 169 & 0 & $0.0 \%$ \\
\hline HUS, total & 15863 & 15688 & 175 & $1.1 \%$ \\
\hline
\end{tabular}


Table 2. Type of incident

Table presents the division of reports among type of incident for non-serious and serious incidents. To ease interpretation, bolded numbers are used for the groups where the percentage of incidents is greater and the difference is statistically significant. ( $n=$ number, $\%=$ percentage of reports compared to group total)

\begin{tabular}{|lcc|cc|r|}
\hline \multicolumn{1}{c}{ Type of incident } & non-serious & \multicolumn{2}{|c|}{ serious } & \multicolumn{2}{l|}{ p value } \\
& $n$ & $\%$ & $n$ & $\%$ & \\
\hline M edication, infusion, blood transfusion, contrast medium & 5900 & $\mathbf{3 7 , 6}$ & 25 & 14,3 & $<0.001$ \\
\hline Information transfer and handling, communication & 3659 & 23,3 & 31 & 17,7 & 0.081 \\
\hline Laboratory, imaging or other tests & 2754 & 17,6 & 41 & $\mathbf{2 3 , 4}$ & 0.043 \\
\hline Other treatment or monitoring & 1282 & 8,2 & 26 & $\mathbf{1 4 , 9}$ & 0.001 \\
\hline Medical equipment or its operation & 880 & 5,6 & 23 & $\mathbf{1 3 , 1}$ & $<0.001$ \\
\hline Accident & 696 & 4,4 & 15 & $\mathbf{8 , 6}$ & 0.009 \\
\hline Other & 637 & 4,1 & 12 & 6,9 & 0.063 \\
\hline Violence & 311 & 2,0 & 11 & $\mathbf{6 , 3}$ & $<0.001$ \\
\hline Asepsis/hygiene & 264 & 1,7 & 1 & 0,6 & N/A \\
\hline Physical surroundings & 233 & 1,5 & 3 & 1,7 & N/A \\
\hline Invasive procedure & 214 & 1,4 & 10 & $\mathbf{5 , 7}$ & $<0.001$ \\
\hline Surgical operation & 174 & 1,1 & 6 & 3,4 & N/A \\
\hline Diagnosis & 101 & 0,6 & 4 & 2,3 & N/A \\
\hline Radiotherapy & 74 & 0,5 & 0 & 0,0 & N/A \\
\hline Not known & 32 & 0,2 & 0 & 0,0 & N/A \\
\hline First aid environment & 16 & 0,1 & 0 & 0,0 & N/A \\
\hline
\end{tabular}


Table 3. Contributing factors

Table presents the division of reports among contributing factors for non-serious and serious incidents. To ease interpretation, bolded numbers are used for the groups where the percentage of incidents is greater and the difference is statistically significant. ( $n=$ number, $\%=$ percentage of reports compared to group total)

\begin{tabular}{|lcc|cc|r|}
\hline & non-serious & \multicolumn{2}{c|}{ serious } & p value \\
& $\mathrm{n}$ & $\%$ & $\mathrm{n}$ & $\%$ & \\
\hline Not known & 4001 & $\mathbf{2 5 , 5}$ & 15 & 8,6 & $<0.001$ \\
\hline Handling of procedures & 2985 & 19,0 & 62 & $\mathbf{3 5 , 4}$ & $<0.001$ \\
\hline Communication and information transfer & 2772 & 17,7 & 28 & 16,0 & 0.564 \\
\hline No contributing factors, normal situation & 2181 & 13,9 & 23 & 13,1 & 0.773 \\
\hline Environment, facilities, resources & 1777 & 11,3 & 19 & 10,9 & 0.845 \\
\hline Training, orientation and skills & 1212 & 7,7 & 27 & $\mathbf{1 5 , 4}$ & $<0.001$ \\
\hline Patient and relatives & 665 & 4,2 & 17 & $\mathbf{9 , 7}$ & $<0.001$ \\
\hline Medical device and equipment & 601 & 3,8 & 23 & $\mathbf{1 3 , 1}$ & $<0.001$ \\
\hline Teamwork & 489 & 3,1 & 18 & $\mathbf{1 0 , 3}$ & $<0.001$ \\
\hline Organization, management & 121 & 0,8 & 12 & $\mathbf{6 , 9}$ & $<0.001$ \\
\hline Medication & 81 & 0,5 & 3 & 1,7 & N/A \\
\hline
\end{tabular}


Figure 1.

Risk Matrix Used with HaiPro Analysis

\begin{tabular}{|c|c|c|c|}
\hline & \multicolumn{3}{|c|}{ HaiPro risk matrix [translation from Finnish by author] } \\
\hline & & Consequences & \\
\hline Likelihood & $\begin{array}{l}\text { Minor } \\
\text { Discomfort, delayed or } \\
\text { prolonged treatment } \\
\text { without substantial } \\
\text { health effects }\end{array}$ & $\begin{array}{l}\text { Major } \\
\text { Health effects requiring } \\
\text { treatment, prolonged } \\
\text { treatment, temporary } \\
\text { incapacity for work }\end{array}$ & $\begin{array}{l}\text { Severe } \\
\text { Death, permanent or } \\
\text { serious harm, } \\
\text { permanent incapacity } \\
\text { for work }\end{array}$ \\
\hline $\begin{array}{l}\text { Remote } \\
\text { random event, short } \\
\text { time exposure }\end{array}$ & I Negligible risk & II Low risk & III Moderate risk \\
\hline $\begin{array}{l}\text { Probable } \\
\text { Near miss events } \\
\text { have happened }\end{array}$ & II Low risk & III Moderate risk & IV High risk \\
\hline $\begin{array}{l}\text { Frequent } \\
\text { Incidents have } \\
\text { happened, near miss } \\
\text { events happen } \\
\text { frequently }\end{array}$ & III Moderate risk & IV High risk & V Serious risk \\
\hline
\end{tabular}


Figure 2.

\section{Handling of a Safety Incident Report in the HaiPro System}

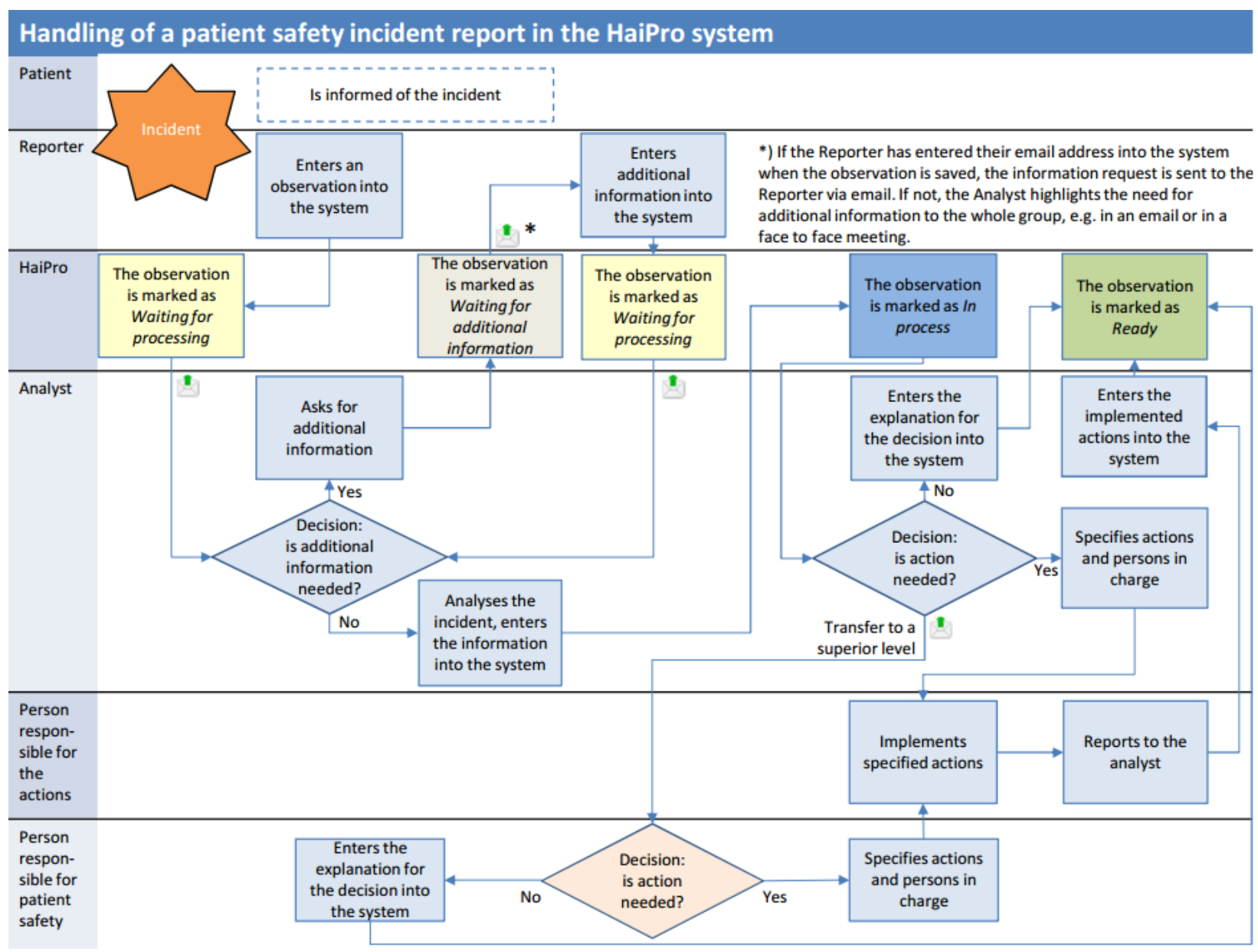

